\title{
SOIL ASSESSMENT QUALITY OF ARABICA COFFEE IN AIE DINGIN, LEMBAH GUMANTI FOR SUSTAINABLE AGRICULTURE
}

\author{
Endar Hidayat $^{{ }^{*} \text {, } \text { Irwan Darfis }^{2} \text {, Yuzirwan Rasyid }}{ }^{2}$, Gusmini $^{2}$ and Hiroyuki Harada ${ }^{1}$ \\ ${ }^{1}$ Department of Environmental Science, Prefectural University of Hiroshima, Shobara, Japan \\ ${ }^{2}$ Department of Soil Science, University of Andalas, Padang, Indonesia \\ https://doi.org/10.35410/IJAEB.2020.5589
}

\begin{abstract}
Arabica coffee is one of the main crop in Aie Dingin, Lembah Gumanti. However, production of arabica coffee in Aie Dingin is still low. Thus, this is important for analysis and assessment soil quality which have related to production of arabica coffee. The present study was conducted in Aie Dingin, Lembah Gumanti. The composite sample were collected from 0-20 cm, 20-40 cm, and $40-60 \mathrm{~cm}$ of soil depth in 12 location. The analysis of soil included: soil $\mathrm{pH}$, cation exchange capacity (CEC), organic matter (OM), and base saturation (BS). The results showed that the most area in acidity, cation exchange capacity (CEC), organic matter (OM), and base saturation (BS) are dominant very low. For improving soil quality, we suggestion adding lime and fertilzer also growing protecting crops for prevent high rainwater directly to land field so that leaching was not occurred.
\end{abstract}

Keywords: Coffee, soil quality, Aie dingin, coffee production, soil nutrient.

\section{INTRODUCTION}

Coffee is one of the most important agricultural commodities in Indonesia. The contribution of this crop have influence in economic sectors. The mainly exported coffee to Japan, India, USA, Singapore, and China [1]. Generally, production of coffee in West Sumatera Province (2413 tons) with total area (8337 ha) in 2019 [2,3].

Arabica coffee is one the most populer coffee in the world which cause the tasted is suitable for many people and high price. Aie dingin is located in highland of West Sumatera Province which have elevation more than $1000 \mathrm{~m}$ asl, it might be suitable for growing arabica coffee. However, production of arabica coffee in Aie Dingin is still low with $450 \mathrm{~kg} / \mathrm{ha} / \mathrm{year}$ [4]. According to [5] that production of arabica coffee per year can be reach 1.8 tons/ha. Thus, this is important for finding the problem for increasing of productivity.

Soil nutrient is the main important factors influence to the productivity of arabica coffee [6]. Aciditity, cation exchange capacity, organic matter is the problem in soil fertility. On the other hand, abiotic factors also influence to the soil quality such as rainwater. So, this study was carried out to assessment soil quality in area of arabica coffee plantation. This is important information to coffee farmers for increasing production of arabica coffee in Aie Dingin, Lembah Gumanti. 
Vol. 5, No. 06; 2020

ISSN: $2456-8643$

\section{MATERIALS AND METHOD}

\subsection{Location and soil sample}

The Aie Dingin has an area of 373.800 ha. It is located between $0^{0} 32$ ' 14 " to $01^{0} 46^{\prime} 45^{\prime \prime}$ (latitudes) and $100^{\circ} 25^{\prime} 00^{\prime \prime}$ to $101^{\circ} 41^{\prime} 41^{\prime \prime}$ (longitudes) which elevation from 1200 to $2300 \mathrm{~m}$ asl with temperature between $18^{\circ} \mathrm{C}$ and $20^{\circ} \mathrm{C}$ and annual rainfall $2333.70 \mathrm{~mm} /$ year [4]. The soil samples is taken by composite were collected from $0-20 \mathrm{~cm}, 20-40 \mathrm{~cm}$, and $40-60 \mathrm{~cm}$ of soil depth in 12 location.

\subsection{Chemical analysis}

The $\mathrm{pH}$ was measured a ratio 1:5 by following [7]. Organic matter was measured by Walkley and Black method [8]. Cation exchange capacity (CEC) were extracted 1M NH4OAc $\mathrm{pH} 7 \mathrm{using}$ atomic absorption spectrofotometer [9]. Base saturation was calculated in Eq (1):

Base saturation $(\%)=\frac{\text { Exchangable Cations }\left(\mathrm{Ca}, \mathrm{Mg}, K_{2} N a\right)}{\text { Cation exchange capacity }(\mathrm{CEC})} \times 100$

\section{RESULTS AND DISCUSSION}

(Table 1) shows that the $\mathrm{pH}$ value which range between 4.70 and 7.5 with the criteria from slightly acid, acid and neutral. The change $\mathrm{pH}$ level in soil depth due to washing process and the accumulation of soil materials by rainwater [10]. The highest volume of rainwater received in soil would be might to become acid might cause increasing hydrogen ions (H+). Low $\mathrm{pH}$ values requiring liming in soil [11]. Soil organic matter is a source of organic compounds that it can be absorbed by plants. Organic matter in the soil is one of the factors that plays a role in the success of agricultural sectors. This is might cause organic matter can improve soil fertility such as physically, chemically, and biology. (Table 2) shows that the most dominant criteria of organic matter are between very low and low, this might be due to high rainfall. Rainfall is the problem in tropical climates and highland. The high rainfall might be influence degradation of organic matter into humus is quickly and then shaping to become soil nutrient. However, the high rainfall also influence soil nutrient is easy to leach into water surface and make poor organic matter that utilize on coffee plant.

Cation exchange capacity (CEC) is the ability or capacity of soil colloids to absorb cations because they are held negatively charged on clay and humus. As shown in (Table 3), the criteria of CEC value in the range very low to low. Soil with low CEC, indicate they receive large of volume of rainwater to leach some of ionic elements in the horizon. As mentioned by Dai et al. that CEC have significant effect with organic matter (OM) due to influence of soil chemical and physical properties [12]. Base saturation is the result of a comparison between base cations and CEC values. Base saturation are generally a nutrient required by plants and have influence to climate, organic matter and soil pH. As shown in (Table 4), the value of base saturation in the range from $9.59 \%$ to $34.01 \%$ with criteria between low to very low. This value might be due to rainwater received is higher. The higher amount of base saturation indicates the more acidity and short time to become neutral conditions [13]. 
International Journal of Agriculture, Environment and Bioresearch

Vol. 5, No. 06; 2020

ISSN: $2456-8643$

Table 1. Soil pH in Aie Dingin, Lembah Gumanti

\begin{tabular}{|c|c|c|c|}
\hline Location & $\operatorname{Depth}(\mathrm{cm})$ & Soil pH & Criteria $^{14}$ \\
\hline \multirow{3}{*}{ Aie Dingin $/ 100^{0} 47^{\prime} 2^{\prime \prime} \mathrm{E} / \mathbf{1}^{0}-7^{\prime}-27^{\prime \prime}$} & $0-20$ & 5.58 & Slightly Acid \\
\hline & $20-40$ & 5.23 & Acid \\
\hline & $40-60$ & 4.99 & Acid \\
\hline \multirow{3}{*}{ Aie Dingin $/ 100^{\circ} 46^{\prime} 45^{\prime \prime} \mathrm{E} / 1^{0}-7^{\prime}-16^{\prime \prime} \mathrm{S}$} & $0-20$ & 5.00 & Acid \\
\hline & $20-40$ & 4.70 & Acid \\
\hline & $40-60$ & 4.70 & Acid \\
\hline \multirow{3}{*}{ Aie Dingin $/ 100^{\circ} 47^{\prime} 4^{\prime \prime} \mathrm{E} /$ - $^{0}-7^{\prime}-29^{\prime \prime} \mathrm{S}$} & $0-20$ & 4.95 & Acid \\
\hline & $20-40$ & 4.95 & Acid \\
\hline & $40-60$ & 5.10 & Acid \\
\hline \multirow{3}{*}{ Aie Dingin $/ 100^{\circ} 47^{\prime} 18^{\prime \prime} \mathrm{E} / 1^{0}-7^{\prime}-17^{\prime \prime} \mathrm{S}$} & $0-20$ & 5.01 & Acid \\
\hline & $20-40$ & 5.01 & Acid \\
\hline & $40-60$ & 5.33 & Acid \\
\hline \multirow{3}{*}{ Aie Dingin $100^{0} 47^{\prime} 23^{\prime \prime} \mathrm{E} /$-1 $^{0}-9^{\prime}-1^{\prime \prime} \mathrm{S}$} & $0-20$ & 7.5 & Neutral \\
\hline & $20-40$ & 7.35 & Neutral \\
\hline & $40-60$ & 6.95 & Neutral \\
\hline \multirow{3}{*}{ Aie Dingin $/ 100^{0} 47^{\prime} 24^{\prime \prime} \mathrm{E} /$ - $^{0}-9^{\prime}-4^{\prime \prime} \mathrm{S}$} & $0-20$ & 5.36 & Acid \\
\hline & $20-40$ & 5.34 & Acid \\
\hline & $40-60$ & 5.59 & Acid \\
\hline \multirow{3}{*}{ Aie Dingin $/ 100^{\circ} 46^{\prime} 26^{\prime \prime} \mathrm{E} / 1^{0}-7^{\prime}-27^{\prime \prime} \mathrm{S}$} & $0-20$ & 7.30 & Neutral \\
\hline & $20-40$ & 7.23 & Neutral \\
\hline & $40-60$ & 7.21 & Neutral \\
\hline \multirow{3}{*}{ Aie Dingin $/ 100^{0} 46^{\prime} 28^{\prime \prime} \mathrm{E} / 1^{0}-7^{\prime}-27^{\prime \prime} \mathrm{S}$} & $0-20$ & 6.01 & Slightly Acid \\
\hline & $20-40$ & 6.45 & Slightly Acid \\
\hline & $40-60$ & 6.46 & Slightly Acid \\
\hline \multirow{3}{*}{ Aie Dingin $/ 100^{\circ} 49^{\prime} 21^{\prime \prime} \mathrm{E} / \mathrm{-}^{0}-11^{\prime}-10^{\prime \prime} \mathrm{S}$} & $0-20$ & 5.94 & Slightly Acid \\
\hline & $20-40$ & 6.16 & Slightly Acid \\
\hline & $40-60$ & 6.23 & Slightly Acid \\
\hline \multirow{3}{*}{ Aie Dingin $/ 100^{\circ} 49^{\prime} 20^{\prime \prime} \mathrm{E} / 1^{0}-11^{\prime}-6{ }^{\prime \prime} \mathrm{S}$} & $0-20$ & 6.39 & Slightly Acid \\
\hline & $20-40$ & 6.35 & Slightly Acid \\
\hline & $40-60$ & 6.12 & Slightly Acid \\
\hline \multirow{3}{*}{ Aie Dingin/100050’39”'E/-1 $1^{0}-11^{\prime} 0^{\prime} \mathrm{S}$} & $0-20$ & 6.68 & Neutral \\
\hline & $20-40$ & 6.60 & Neutral \\
\hline & $40-60$ & 6.76 & Neutral \\
\hline \multirow{3}{*}{ Aie Dingin $/ 100^{0} 50^{\prime} 40^{\prime \prime} \mathrm{E} / 1^{0}-11^{\prime}-1{ }^{\prime \prime} \mathrm{S}$} & $0-20$ & 6.79 & Neutral \\
\hline & $20-40$ & 6.23 & Slightly Acid \\
\hline & $40-60$ & 6.89 & Neutral \\
\hline
\end{tabular}


International Journal of Agriculture, Environment and Bioresearch

Vol. 5, No. 06; 2020

ISSN: $2456-8643$

Table 2. Soil organic matter in Aie Dingin, Lembah Gumanti

\begin{tabular}{|c|c|c|c|}
\hline Location & Depth(cm) & Organic matter (\%) & Criteria $^{14}$ \\
\hline \multirow{3}{*}{$\begin{array}{c}\text { Aie Dingin } / 100^{0} 47^{\prime} 2^{\prime \prime} \mathrm{E} / \mathrm{-}^{0}{ }^{-} \\
7^{\prime}-27^{\prime \prime}\end{array}$} & $0-20$ & 0.33 & Very Low \\
\hline & $20-40$ & 0.28 & Very Low \\
\hline & $40-60$ & 0.14 & Very Low \\
\hline \multirow{3}{*}{$\begin{array}{c}\text { Aie Dingin } / 100^{0} 46^{\prime} 45^{\prime \prime} \mathrm{E} / \mathrm{-1}^{0}{ }_{-} \\
7^{\prime}-16^{\prime} \mathrm{S}\end{array}$} & $0-20$ & 0.16 & Very Low \\
\hline & $20-40$ & 0.24 & Very Low \\
\hline & $40-60$ & 0.10 & Very Low \\
\hline \multirow{3}{*}{$\begin{array}{c}\text { Aie Dingin } / 100^{0} 47^{\prime} 4^{\prime \prime} \mathrm{E} / \mathrm{-}^{0}{ }^{-} \\
7^{\prime}-29^{\prime \prime} \mathrm{S}\end{array}$} & $0-20$ & 0.77 & Very Low \\
\hline & $20-40$ & 0.34 & Very Low \\
\hline & $40-60$ & 0.45 & Very Low \\
\hline \multirow{3}{*}{$\begin{array}{c}\text { Aie Dingin } / 100^{0} 47^{\prime} 18^{\prime \prime} \mathrm{E} / \mathrm{-1}^{0}{ }_{-} \\
7^{\prime}-17^{\prime} \mathrm{S}\end{array}$} & $0-20$ & 1.18 & Low \\
\hline & $20-40$ & 0.61 & Very Low \\
\hline & $40-60$ & 0.17 & Very Low \\
\hline \multirow{3}{*}{$\begin{array}{c}\text { Aie Dingin } / 100^{0} 47^{\prime} 23^{\prime \prime} \mathrm{E} / \mathrm{-}^{0}{ }^{-} \\
\text {9'-1"S }\end{array}$} & $0-20$ & 0.80 & Very Low \\
\hline & $20-40$ & 0.82 & Very Low \\
\hline & $40-60$ & 0.61 & Very Low \\
\hline \multirow{3}{*}{$\begin{array}{c}\text { Aie Dingin } / 100^{0} 47^{\prime} 24^{\prime \prime} \mathrm{E} / \mathrm{-}^{0}{ }^{-} \\
\text {9'-4"S }\end{array}$} & $0-20$ & 1.03 & Low \\
\hline & $20-40$ & 0.90 & Very Low \\
\hline & $40-60$ & 0.73 & Very Low \\
\hline \multirow{3}{*}{$\begin{array}{c}\text { Aie Dingin } / 100^{0} 466^{\prime} 26^{\prime \prime} \mathrm{E} / \mathbf{1}^{0}{ }^{-} \\
7^{\prime}-27^{\prime} \mathrm{S}\end{array}$} & $0-20$ & 0.51 & Very Low \\
\hline & $20-40$ & 0.38 & Very Low \\
\hline & $40-60$ & 0.21 & Very Low \\
\hline \multirow{2}{*}{$\begin{array}{c}\text { Aie Dingin } / 100^{0} 46^{\prime} 28^{\prime \prime} \mathrm{E} / \mathrm{-1}^{0}{ }^{-} \\
7^{\prime}-27^{\prime} \mathrm{S}\end{array}$} & $0-20$ & 0.24 & Very Low \\
\hline & $20-40$ & 0.07 & Very Low \\
\hline
\end{tabular}


International Journal of Agriculture, Environment and Bioresearch

Vol. 5, No. 06; 2020

ISSN: $2456-8643$

\begin{tabular}{|c|c|c|c|}
\hline & $40-60$ & 0.04 & Very Low \\
\hline \multirow{3}{*}{$\begin{array}{c}\text { Aie Dingin } / 100^{0} 49^{\prime} 21^{\prime \prime} \mathrm{E} / \mathrm{-}^{0}{ }^{-} \\
11^{\prime}-10^{\prime \prime} \mathrm{S}\end{array}$} & $0-20$ & 0.33 & Very Low \\
\hline & $20-40$ & 0.28 & Very Low \\
\hline & $40-60$ & 0.14 & Very Low \\
\hline \multirow{3}{*}{$\begin{array}{c}\text { Aie Dingin } / 100^{0} 49^{\prime} 20^{\prime \prime} \mathrm{E} / \mathrm{-}^{0}{ }_{-} \\
11^{\prime}-6^{\prime \prime} \mathrm{S}\end{array}$} & $0-20$ & 0.16 & Very Low \\
\hline & $20-40$ & 0.24 & Very Low \\
\hline & $40-60$ & 0.10 & Very Low \\
\hline \multirow{3}{*}{$\begin{array}{c}\text { Aie Dingin } / 100^{0} 50^{\prime} 39^{\prime \prime} \mathrm{E} /-^{0}{ }^{\circ} \\
11^{\prime} 0^{\prime} \mathrm{S}\end{array}$} & $0-20$ & 0.29 & Very Low \\
\hline & $20-40$ & 0.13 & Very Low \\
\hline & $40-60$ & 0.19 & Very Low \\
\hline \multirow{3}{*}{$\begin{array}{c}\text { Aie Dingin } / 100^{0} 50^{\prime} 40^{\prime \prime} \mathrm{E} / \mathrm{-}^{0}{ }_{-} \\
11^{\prime}-1{ }^{\prime \prime} \mathrm{S}\end{array}$} & $0-20$ & 1.07 & Low \\
\hline & $20-40$ & 0.84 & Very Low \\
\hline & $40-60$ & 0.74 & Very Low \\
\hline
\end{tabular}

Table 3. Soil cation exchange capacity (CEC) in Aie Dingin, Lembah Gumanti

\begin{tabular}{|c|c|c|c|}
\hline Location & Depth (cm) & CEC (me/100g) & Criteria $^{14}$ \\
\hline \multirow{3}{*}{ Aie Dingin $/ 100^{0} 47^{\prime} 2^{\prime \prime} \mathrm{E} / 1^{0}-7^{\prime}-27^{\prime \prime}$} & $0-20$ & 7.57 & Low \\
\hline & $20-40$ & 5.43 & Low \\
\hline & $40-60$ & 4.14 & Very Low \\
\hline \multirow{3}{*}{$\begin{array}{c}\text { Aie Dingin } / 100^{0} 46^{\prime} 45^{\prime \prime} \mathrm{E} /-^{0}{ }^{0} 7^{\prime}- \\
16^{\prime \prime} \mathrm{S}\end{array}$} & $0-20$ & 6.96 & Low \\
\hline & $20-40$ & 9.56 & Low \\
\hline & $40-60$ & 11.25 & Low \\
\hline \multirow{3}{*}{$\begin{array}{c}\text { Aie Dingin } / 100^{0} 47^{\prime} 4^{\prime \prime} \mathrm{E} / \mathrm{-}^{0}{ }^{0} 7^{\prime}- \\
29^{\prime} \mathrm{S}\end{array}$} & $0-20$ & 9.56 & Low \\
\hline & $20-40$ & 9.38 & Low \\
\hline & $40-60$ & 9.27 & Low \\
\hline
\end{tabular}


International Journal of Agriculture, Environment and Bioresearch

Vol. 5, No. 06; 2020

ISSN: $2456-8643$

\begin{tabular}{|c|c|c|c|}
\hline \multirow{3}{*}{$\begin{array}{c}\text { Aie Dingin } / 100^{0} 47^{\prime} 18^{\prime \prime} \mathrm{E} / \mathrm{-}^{0}-7^{\prime}- \\
17^{\prime} \mathrm{S}\end{array}$} & $0-20$ & 8.95 & Low \\
\hline & $20-40$ & 6.41 & Low \\
\hline & $40-60$ & 4.48 & Very Low \\
\hline \multirow{3}{*}{$\begin{array}{c}\text { Aie Dingin } / 100^{0} 47^{\prime} 23^{\prime \prime} \mathrm{E} / \mathbf{1}^{0}-9^{\prime}- \\
1 \text { 'S }\end{array}$} & $0-20$ & 11.27 & Low \\
\hline & $20-40$ & 4.30 & Very Low \\
\hline & $40-60$ & 10.54 & Low \\
\hline \multirow{3}{*}{$\begin{array}{c}\text { Aie Dingin } / 100^{0} 47^{\prime} 24^{\prime \prime} \mathrm{E} / \text { - }^{0}-9^{\prime}- \\
4 ” \mathrm{~S}\end{array}$} & $0-20$ & 11.98 & Low \\
\hline & $20-40$ & 13.16 & Low \\
\hline & $40-60$ & 10.06 & Low \\
\hline \multirow{3}{*}{$\begin{array}{c}\text { Aie Dingin/ } 100^{0} 46{ }^{\prime} 26^{\prime} \mathrm{E} / \mathrm{-}^{0}-7^{\prime}- \\
27^{\prime} \mathrm{S}\end{array}$} & $0-20$ & 9.33 & Low \\
\hline & $20-40$ & 6.24 & Low \\
\hline & $40-60$ & 3.46 & Very Low \\
\hline \multirow{3}{*}{$\begin{array}{c}\text { Aie Dingin } / 100^{0} 46^{\prime} 28^{\prime \prime} \mathrm{E} / \mathbf{1}^{0}-7^{\prime}- \\
27^{\prime} \mathrm{S}\end{array}$} & $0-20$ & 5.92 & Low \\
\hline & $20-40$ & 10.87 & Low \\
\hline & $40-60$ & 6.15 & Low \\
\hline \multirow{3}{*}{$\begin{array}{c}\text { Aie Dingin } / 100^{\circ} 49^{\prime} 21^{\prime \prime} \mathrm{E} / \mathrm{-}^{0}{ }^{\circ}-11^{\prime}- \\
10^{\prime} \mathrm{S}\end{array}$} & $0-20$ & 8.53 & Low \\
\hline & $20-40$ & 7.11 & Low \\
\hline & $40-60$ & 6.02 & Low \\
\hline \multirow{3}{*}{$\begin{array}{c}\text { Aie Dingin } / 100^{\circ} 49^{\prime} 20^{\prime \prime} \mathrm{E} / \mathrm{-1}^{0}-11^{\prime}- \\
6{ }^{\prime} \mathrm{S}\end{array}$} & $0-20$ & 4.67 & Very Low \\
\hline & $20-40$ & 3.40 & Very Low \\
\hline & $40-60$ & 3.57 & Very Low \\
\hline \multirow{3}{*}{$\begin{array}{c}\text { Aie Dingin } / 100^{0} 50^{\prime} 39^{\prime \prime} \mathrm{E} /-^{0}{ }^{-} \\
11^{\prime} 0^{\prime} \mathrm{S}\end{array}$} & $0-20$ & 4.41 & Very Low \\
\hline & $20-40$ & 2.83 & Very Low \\
\hline & $40-60$ & 9.24 & Low \\
\hline Aie Dingin $/ 100^{0} 50^{\prime} 40^{\prime \prime} \mathrm{E} / \mathrm{-}^{0}{ }^{-}-11^{\prime}-$ & $0-20$ & 5.45 & Low \\
\hline
\end{tabular}


International Journal of Agriculture, Environment and Bioresearch

Vol. 5, No. 06; 2020

ISSN: $2456-8643$

\begin{tabular}{|c|c|c|c|}
\hline \multirow{2}{*}{1 "S } & $20-40$ & 8.84 & Low \\
\cline { 2 - 4 } & $40-60$ & 6.63 & Low \\
\hline
\end{tabular}

Table 4. Soil base Saturation (BS) in Aie Dingin, Lembah Gumanti

\begin{tabular}{|c|c|c|c|}
\hline Location & Depth(cm) & Base Saturation(\%) & Criteria $^{14}$ \\
\hline \multirow{3}{*}{$\begin{array}{c}\text { Aie Dingin } / 100^{0} 47^{\prime} 2^{\prime \prime} \mathrm{E} / \mathbf{- 1}^{0}- \\
7^{\prime}-27^{\prime \prime}\end{array}$} & $0-20$ & 9.59 & Very Low \\
\hline & $20-40$ & 15.96 & Very Low \\
\hline & $40-60$ & 19.30 & Very Low \\
\hline \multirow{3}{*}{$\begin{array}{c}\text { Aie Dingin } / 100^{0} 46^{\prime} 45^{\prime \prime} \mathrm{E} / \mathrm{-1}^{0}{ }_{-} \\
7^{\prime}-16^{\prime} \mathrm{S}\end{array}$} & $0-20$ & 20.09 & Very Low \\
\hline & $20-40$ & 11.36 & Very Low \\
\hline & $40-60$ & 13.06 & Very Low \\
\hline \multirow{3}{*}{$\begin{array}{c}\text { Aie Dingin } / 100^{0} 47^{\prime} 4^{\prime \prime} \mathrm{E} / \mathrm{-}^{0}{ }^{-} \\
7^{\prime}-29^{\prime} \mathrm{S}\end{array}$} & $0-20$ & 11.39 & Very Low \\
\hline & $20-40$ & 12.29 & Very Low \\
\hline & $40-60$ & 10.57 & Very Low \\
\hline \multirow{3}{*}{$\begin{array}{c}\text { Aie Dingin } / 100^{0} 47^{\prime} 18^{\prime \prime} \mathrm{E} / \mathrm{-1}^{0}{ }_{-} \\
7^{\prime}-17^{\prime \prime} \mathrm{S}\end{array}$} & $0-20$ & 13.79 & Very Low \\
\hline & $20-40$ & 18.13 & Very Low \\
\hline & $40-60$ & 21.08 & Very Low \\
\hline \multirow{3}{*}{$\begin{array}{c}\text { Aie Dingin } / 100^{0} 47^{\prime} 23^{\prime \prime} \mathrm{E} / \mathrm{-1}^{0}{ }_{-} \\
9^{\prime}-1^{\prime \prime} \mathrm{S}\end{array}$} & $0-20$ & 12.03 & Very Low \\
\hline & $20-40$ & 21.14 & Low \\
\hline & $40-60$ & 10.23 & Very Low \\
\hline \multirow{3}{*}{$\begin{array}{c}\text { Aie Dingin } / 100^{0} 47^{\prime} 24^{\prime \prime} \mathrm{E} / \mathrm{-1}^{0}{ }_{-} \\
9^{\prime}-4^{\prime \prime} \mathrm{S}\end{array}$} & $0-20$ & 14.85 & Very Low \\
\hline & $20-40$ & 13.38 & Very Low \\
\hline & $40-60$ & 12.56 & Very Low \\
\hline \multirow{3}{*}{$\begin{array}{c}\text { Aie Dingin } / 100^{0} 46^{\prime} 26^{\prime \prime} \mathrm{E} / \mathbf{1}^{0}{ }^{-} \\
7^{\prime}-27^{\prime \prime} \mathrm{S}\end{array}$} & $0-20$ & 11.38 & Very Low \\
\hline & $20-40$ & 19.39 & Very Low \\
\hline & $40-60$ & 34.01 & Low \\
\hline
\end{tabular}


International Journal of Agriculture, Environment and Bioresearch

Vol. 5, No. 06; 2020

ISSN: $2456-8643$

\begin{tabular}{|c|c|c|c|}
\hline \multirow{3}{*}{$\begin{array}{c}\text { Aie Dingin } / 100^{0} 46^{\prime} 28^{\prime \prime} \mathrm{E} / \mathrm{-1}^{0}- \\
7^{\prime}-27^{\prime \prime} \mathrm{S}\end{array}$} & $0-20$ & 17.93 & Very Low \\
\hline & $20-40$ & 11.41 & Very Low \\
\hline & $40-60$ & 16.15 & Very Low \\
\hline \multirow{3}{*}{$\begin{array}{c}\text { Aie Dingin } / 100^{0} 49^{\prime} 21^{\prime \prime} \mathrm{E} / \mathrm{-1}^{0}{ }_{-} \\
11^{\prime}-10^{\prime \prime} \mathrm{S}\end{array}$} & $0-20$ & 11.21 & Very Low \\
\hline & $20-40$ & 16.12 & Very Low \\
\hline & $40-60$ & 18.42 & Very Low \\
\hline \multirow{3}{*}{$\begin{array}{c}\text { Aie Dingin } / 100^{\circ} 49^{\prime} 20^{\prime \prime} \mathrm{E} / \mathrm{-}^{0}{ }_{-} \\
11^{\prime}-6^{\prime \prime} \mathrm{S}\end{array}$} & $0-20$ & 15.14 & Very Low \\
\hline & $20-40$ & 19.4 & Very Low \\
\hline & $40-60$ & 18.300 & Very Low \\
\hline \multirow{3}{*}{$\begin{array}{c}\text { Aie Dingin } / 100^{0} 50^{\prime} 39^{\prime \prime} \mathrm{E} / \mathrm{-1}^{0}{ }_{-} \\
11^{\prime} 0^{\prime \prime} \mathrm{S}\end{array}$} & $0-20$ & 17.23 & Very Low \\
\hline & $20-40$ & 20.41 & Low \\
\hline & $40-60$ & 10.12 & Very Low \\
\hline \multirow{3}{*}{$\begin{array}{c}\text { Aie Dingin } / 100^{0} 50^{\prime} 40^{\prime \prime} \mathrm{E} / \mathrm{-1}^{0}- \\
11^{\prime}-1^{\prime \prime} \mathrm{S}\end{array}$} & $0-20$ & 20.67 & Low \\
\hline & $20-40$ & 11.40 & Very Low \\
\hline & $40-60$ & 20.43 & Low \\
\hline
\end{tabular}

\section{CONCLUSION}

This study concluded that soil $\mathrm{pH}$ in the area coffee of Aie Dingin are mostly acidity, it might be required to adding lime for increasing the value. Organic matter $(\mathrm{OM})$, cation exchange capacity (CEC) and base saturation (BS) are mostly very low. This might be due to the high of rainwater volume is received which leaching in horizon. However, for sustainable agriculture we suggestion for growing protecting crops for prevent of rainwater directly to the land field and also requiring fertilizer for increasing nutrient in soil.

\section{Acknowledge}

The author (E.H) would like to thanks to the (Mext Scholarship) for funding while studying in Prefectural University of Hiroshima. 
Vol. 5, No. 06; 2020

ISSN: $2456-8643$

\section{REFERENCE}

1. Y Yusmarni., A Putri., C Paloma., and Z Zakir., Production analysis of smallholding arabica coffee farm in the distict of Solok, West Sumatra, Indonesia. IOP Conference Series; Earth and Environmental Science; 583. 012020. (2020).

2. Statistics of West Sumatera Province (BPS)., Production of smallholder estate. West Sumatera, (2020).

3. Statistics of West Sumatera Province (BPS)., Planted area of smallholder. West Sumatera, (2020).

4. E Hidayat., A Afriliana., G Gusmini., I Darfis., Y Rasyid., and H Harada., Land suitability evaluation of arabica coffee (Coffea arabica L) plantation in Subdistrict Aie Dingin, Lembah Gumanti, Indonesia. Iop Conference Series: Earth Environmental Science, 583, 012005, (2020).

5. Syakir M., Cultivation and harvest coffee. Plantation research and development center. Bogor, (2010).

6. P.A. Núñez., A. Pimentel., I. Almonte., D Sotomayor-Ramírez., N Martínez., A Pérez., and CM Céspedes. Soil fertility evaluation of coffee (Coffea spp.) production systems and management recomendations for the Barahona Province, Dominican Republic. Journal of Soil Sciences and Plant Nutrition, Vol 11(1), Pages; 127-140, (2011).

7. Jones D., Murphy D., Khalid M., Ahmad W., Edwards-Jones G., and DeLuca T, Short-term biochar-induced increase in soil $\mathrm{CO} 2$ release is both biotically and abiotically mediated. Soil Biology and Biochemistry, 43: 1723-1731, (2011).

8. Rayment G.E., and Higginson F.R., Australian laboratory handbook of soil and water chemical methods. Australian soil and land survey handbook. Inkata press, Melbourne, Sydney, 330. (1992).

9. USDA (United States Department of Agriculture)., Soil survey laboratory methods manual. P. 167-365, 616-643. In Burt, R. (Ed). Soil survey investigations report No. 42, Vers. 4.0. Natural resources conservation service, (2004).

10. Foth H.D., and L.M. Turk., Fundamentals of Soil Science, Wiley, New York. (1998).

11. Ayodele F.G., Aina O.A., Agboola K., and Musa M.B., Assessment of soil fertility status of some agricultural land use types ayetoro-gbede ijumu local government area of Kogi State, Nigeraia. East African Scholars Journal of Agriculture and Life Sciences, 2 (9), 433-438. (2019). 